\title{
Single layers and multilayers of GaN and AIN in square-octagon structure: Stability, electronic properties, and functionalization
}

\author{
E. Gürbüz, ${ }^{1}$ S. Cahangirov, ${ }^{2, *}$ E. Durgun, ${ }^{2}$ and S. Ciraci ${ }^{1, \dagger}$ \\ ${ }^{1}$ Department of Physics, Bilkent University, 06800 Ankara, Turkey \\ ${ }^{2}$ UNAM-Institute of Materials Science and Nanotechnology, Bilkent University, 06800 Ankara, Turkey \\ (Received 11 June 2017; revised manuscript received 11 September 2017; published 20 November 2017)
}

\begin{abstract}
Further to planar single-layer hexagonal structures, GaN and AlN can also form free-standing, single-layer structures constructed from squares and octagons. We performed an extensive analysis of dynamical and thermal stability of these structures in terms of $a b$ initio finite-temperature molecular dynamics and phonon calculations together with the analysis of Raman and infrared active modes. These single-layer square-octagon structures of GaN and AlN display directional mechanical properties and have wide, indirect fundamental band gaps, which are smaller than their hexagonal counterparts. These density functional theory band gaps, however, increase and become wider upon correction. Under uniaxial and biaxial tensile strain, the fundamental band gaps decrease and can be closed. The electronic and magnetic properties of these single-layer structures can be modified by adsorption of various adatoms, or by creating neutral cation-anion vacancies. The single-layer structures attain magnetic moment by selected adatoms and neutral vacancies. In particular, localized gap states are strongly dependent on the type of vacancy. The energetics, binding, and resulting electronic structure of bilayer, trilayer, and three-dimensional (3D) layered structures constructed by stacking the single layers are affected by vertical chemical bonds between adjacent layers. In addition to van der Waals interaction, these weak vertical bonds induce buckling in planar geometry and enhance their binding, leading to the formation of stable 3D layered structures. In this respect, these multilayers are intermediate between van der Waals solids and wurtzite crystals, offering a wide range of tunability.
\end{abstract}

DOI: 10.1103/PhysRevB.96.205427

\section{INTRODUCTION}

Three-dimensional (3D) group III-V compound nitride semiconductors in wurtzite and zinc-blende structures, in particular GaN and AlN, have been subjects of active research in materials science and device physics. As wide-band-gap semiconductors, they are used in a wide range of technological applications, in microwave communication, lasers, detectors, light emitting diodes, water/air purification in UV range, etc. [1,2]. After the synthesis of graphene [3], questions have been raised as to whether group IV elemental and group III-V compound semiconductors can form stable single-layer (SL) graphenelike structures, despite the fact that they do not have layered structures like graphite. As early as in 2005, theoretical studies based on the total energy minimization have shown that, in fact silicon, GaN, GaAs, and AlN can form stable, SL structures in honeycomb structure with twodimensional (2D) hexagonal $(h)$ lattice [4]. Motivated with this result, extensive studies based on ab initio density functional theory (DFT) calculations of total energy, phonon, and finitetemperature molecular dynamics calculations demonstrated that SL silicene [4,5], germanene [5], group IV-IV and III-V compounds [4,6-8], as well as group II-VI compounds like $\mathrm{ZnO}$ [9], can form stable, free-standing, SL structures. Now, silicene and germanene can be grown on metal substrates [10-12].

Prediction of free-standing SL GaN and AlN in planar honeycomb structure, i.e., $h$-AlN and $h$-GaN, have initiated several theoretical studies attempting to reveal their mechan-

\footnotetext{
*seymur@unam.bilkent.edu.tr

†ciraci@fen.bilkent.edu.tr
}

ical, electronic, and optical properties [13-27]. While 3D wurtzite $\mathrm{GaN}(w z-\mathrm{GaN})$ is a direct-band-gap semiconductor and corresponds to a global minimum, SL $h$-GaN has relatively larger indirect fundamental band gap [21]. This situation is attributed to the confinement effect. Conversely, while 3D $w z$-AlN has a wide direct band gap, SL $h$-AlN is a relatively smaller but indirect band gap. Additionally, these SL phases also display optical properties different from those of their parent 3D $w z$-structures. Meanwhile, important optoelectronic applications [1] of 3D $w z-\mathrm{GaN}$ and $w z-\mathrm{AlN}$ rendered the synthesis of 2D SL AlN and GaN a priority study. Eight years after its first prediction [4], Tsipas et al. [28] have demonstrated the epitaxial growth of ultrathin hexagonal $h$-AlN on $\mathrm{Ag}(111)$ substrate. More recently, Balushi et al. [29] achieved the growth of one- to two-layer $2 \mathrm{D} h-\mathrm{GaN}$ on $\mathrm{SiC}(0001)$ surface via graphene encapsulation method.

The physical and chemical properties of 2D SL structures have a close bearing on their atomic structure and 2D lattice symmetry. In addition to the planar SL honeycomb structure of carbon, namely graphene, other 2D allotropes, graphyne and graphdynes, were also investigated [30-34]. $\alpha$ - and $\beta$ graphynes are semimetals with Dirac cones, but $\gamma$-graphyne is a semiconductor. Another critical example is SL $h-\mathrm{MoS}_{2}$ that has a honeycomb structure and is a semiconductor, while $\mathrm{SL} \mathrm{MoS}$ structure consisting of square and octagons (or $s o$-structure) have bands showing both massless Dirac fermion and heavy fermion character [33]. It has been shown that pnictogens can also form buckled $s o$-structures with different properties [35]. In view of these findings, possible square-octagon (haeckelite) structures of SL GaN and AIN have been brought into focus. In fact, more recently, SL so-GaN [36,37] and so-AlN [38] structures have been predicted by first-principles calculations. More recently, Kolobov 
et al. [23], based on first-principles calculations, pointed out that a few-layer $h$-GaN gets instability and spontaneously reconstructs into a structure, which is hexagonal in plane, but forms haeckelite structure consisting of alternating square and octagons with bonds between adjacent layers.

Motivated by the diverse properties of 2D SL $h-\mathrm{GaN}$ and $h$-AlN predicted by theoretical studies, also recent synthesis of ultrathin 2D GaN and AlN layers of SL GaN and AlN, we carried out an extensive study on SL and multilayer (ML) so-GaN and so-AlN structures using spin-polarized DFT. First, the dynamical and thermal stability of $s o$-structures of $\mathrm{GaN}$ and AlN are assured by performing ab initio phonon and finite-temperature molecular dynamics calculations. Analysis of phonon modes revealed Raman and infrared (IR) active modes, which constitute firm data to compare with experiment. Calculations of elastic properties, such as in-plane stiffness and Poisson's ratio, present evidence that these SL square-octagon structures are rather robust. The calculated cohesive energies are comparable to those of SL $h-\mathrm{GaN}$ and $h$-AlN, but smaller than those of $3 \mathrm{D} w z-\mathrm{GaN}$ and $w z-\mathrm{AlN}$, implying a negative formation energy. Both $s o-\mathrm{GaN}$ and $s o$-AlN have indirect band gaps smaller than those of SL $h$-GaN and $h$-AlN. Additionally, their gaps decrease and eventually close with increasing uniaxial and biaxial strains. In bilayer (BL), trilayer (TL), and 3D periodic layered structures having diverse stacking geometries, the vertical anion-cation bonds form between layers; they are longer than similar in-plane bonds, but induce chemical interaction in addition to the attractive vdW interaction. This way, modification of physical properties, in particular those of the electronic structure, with the number of layers are enhanced and binding energies between the layers are increased. Finally, we investigated the chemical doping of SL $s o$-GaN and $s o-\mathrm{AlN}$ with selected adatoms and showed that the electronic structure of the bare $s o$-structures can be modified by the localized gap states. On the other hand, the vacancy of a cation-anion pair leads to local deformations and also induces localized gap states as well as permanent magnetic moments.

\section{METHOD}

We have performed self-consistent field, first-principles plane-wave calculations within spin-polarized, density functional theory (DFT) for total energy and electronic structure calculations. The projector-augmented-wave (PAW) formalism [39] is used as implemented in the Vienna $a b$ initio simulation package (VASP) [40,41]. The electron exchange and correlation (XC) potential is described by the PerdewBurke-Ernzerhof (PBE) form within the generalized gradient approximation (GGA) [42]. In the self-consistent field potential and total energy calculations, a $(9 \times 9 \times 1)$ set of $k$ points generated by Monkhorst-Pack scheme [43] is used for Brillouin zone (BZ) integration. Various tests are performed regarding vacuum level, kinetic energy cutoff, number of bands, $k$ points, and grid points. Kinetic energy cutoff for plane-wave basis set is taken as $520 \mathrm{eV}$. The total energies are minimized with an energy difference between the sequential steps set to $10^{-5} \mathrm{eV}$ for convergence. The Fermi level smearing factor was taken as $0.05 \mathrm{eV}$, but $0.01 \mathrm{eV}$ for band structure calculations. All atomic positions and lattice constants are optimized within the conjugate gradient method until the atomic forces were less than $0.002 \mathrm{eV} / \AA$. Pressures on the lattice unit cell are decreased to values less than $1 \mathrm{kB}$. Vacuum spacing is kept at $15 \AA$ between single layers.

In analyzing energetics, the cohesive energies of SL $s o-G a N$ and $s o$-AlN structures (per a pair of anion $(A=\mathrm{N})$ and cation $(C=\mathrm{Ga}$ or $\mathrm{Al})$ or $C-A)$ are calculated using the expression $E_{c}[\mathrm{Ga}(\mathrm{Al}) \mathrm{N}]=E_{T}[\mathrm{Ga}(\mathrm{Al})]+E_{T}[\mathrm{~N}]-E_{T}[\mathrm{Ga}(\mathrm{Al}) \mathrm{N}]$, where $E_{T}[\mathrm{Ga}(\mathrm{Al})]$ and $E_{T}[\mathrm{~N}]$ are the total energies of free $\mathrm{Ga}(\mathrm{Al})$ and $\mathrm{N}$ atoms, respectively, and $E_{T}[\mathrm{Ga}(\mathrm{Al}) \mathrm{N}]$ is the total energy of $\mathrm{SL} s o-\mathrm{Ga}(\mathrm{Al}) \mathrm{N}$ structure per $C-A$ pair. By definition, the binding structures have $E_{c}>0$. The formation energy of a SL $s o-\mathrm{Ga}(\mathrm{Al}) \mathrm{N}$ structure at $T=0 \mathrm{~K}$, $E_{f}[\mathrm{Ga}(\mathrm{Al}) \mathrm{N}]=E_{c}[\mathrm{Ga}(\mathrm{Al}) \mathrm{N}]-E_{c}[w z-\mathrm{Ga}(\mathrm{Al}) \mathrm{N}]$ is calculated by subtracting the cohesive energy of bulk $w z$-crystal from that of corresponding SL $s o$-structure. Generally, $E_{f}<0$ indicates that the corresponding structure may correspond to a local minimum on the Born-Oppenheimer surface. The average binding energy of layers in a ML structure consisting of $n$ layers can be expressed as $E_{l b}=\left\{n E_{T}[\mathrm{Ga}(\mathrm{Al}) \mathrm{N}]-E_{T}[\mathrm{ML}\right.$ $\mathrm{Ga}(\mathrm{Al}) \mathrm{N}]\} / n$ (per $C-A$ pair) relative to constituent SL $s o-\mathrm{GaN}$ or $s o-\mathrm{AlN}$.

We calculate the phonon spectrum using a small displacement method. The force constant matrix is constructed by slight displacement of atoms in a $(6 \times 6 \times 1)$ supercell. PHON software was used to determine the necessary displacements and to calculate the phonon dispersions using the obtained force constants [44].

\section{SQUARE-OCTAGON STRUCTURES AND THEIR STABILITIES}

We first determine the optimized structures of SL $s o-G a N$ and $s o$-AlN as described in Fig. 1(a). They are planar and have 2D square lattice. In Fig. 1(b), isosurfaces and contour plots of charge density describe cation-anion bonds and charge transfer from cations to anions. The total energies of SL $s o-\mathrm{GaN}$ and $s o-\mathrm{AlN}$ without $\mathrm{vdW}$ correction are calculated to

(a)



(b) $a_{1}=a_{2}=6.42 \AA$

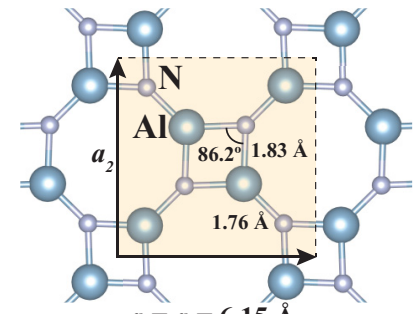
$a_{1}=a_{2}=6.15 \AA$
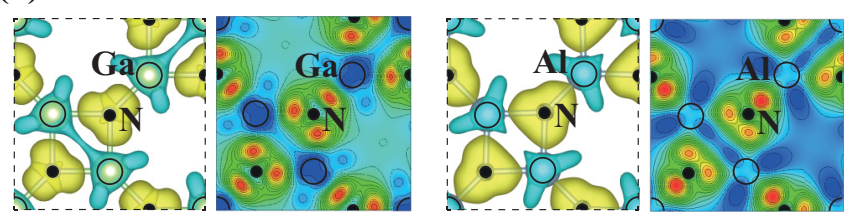

FIG. 1. (a) Atomic configuration of SL planar so-GaN and soAlN. Large green, large blue, and small gray balls are, respectively, $\mathrm{Ga}, \mathrm{Al}$, and $\mathrm{N}$ atoms. Square unit cells are delineated by Bravais lattice vectors. Calculated values of two different cation-anion bonds (long ones $d_{1}$, short ones $d_{2}$ ) are indicated. (b) Charge density isosorfaces and contour plots of SL planar so-GaN and so-AlN. Charge densities increase from dark blue-green-yellow-red. 
be -10.93 and $-13.48 \mathrm{eV}$ per cation-anion pair, respectively. These energies change to -11.08 and $-13.63 \mathrm{eV}$ per $C-A$ pair after vdW correction. Calculated values (without vdW correction) of structural parameters, cohesive and formation energies, elastic constants, and band gaps of SL $s o-\mathrm{GaN}$ and $s o$-AlN are presented in Tables I and II. In the same tables, those values of $h$-GaN, $h$-AlN, 3D $w z-\mathrm{GaN}$, and $3 \mathrm{D} w z-\mathrm{AlN}$ are also included for the sake of comparison. Cation-anion bonds of two different lengths, namely, bonds of squares $\left(d_{1}\right)$ and bonds of octagons $\left(d_{2}\right)$, are distinguished. As compared to SL planar $h$-GaN and $h$-AlN, so-structures are open structures with relatively smaller atom density per unit area and have cohesive energies of 7.53 and $10.05 \mathrm{eV}$ per Ga-N and Al-N pair, respectively. However, their formation energies $\left(E_{f}=-1.23 \mathrm{eV}\right.$ for $s o-\mathrm{GaN}$ and $E_{f}=-2.23 \mathrm{eV}$ for $s o-\mathrm{AlN}$ ) are found to be negative and indicate that these structures may correspond to local minima. The stability in these local minima are assured through the ab initio calculation of phonon frequencies, high-temperature molecular dynamics, and elastic constants.

\section{A. Dynamical stability, symmetries of phonons}

Since optimized SL so-GaN and so-AlN have sizable positive cohesive, but negative, formation energy, the tests of stability of these structures become crucial. Dynamical stability at $T=0 \mathrm{~K}$ is tested by ab initio phonon calculation in the Brillouin zone [44]. Phonon dispersions along symmetry directions of the BZ are presented in Fig. 2(a). Positive frequencies calculated for all $\mathbf{k}$ points ensure the dynamical stability of SL $s o$-GaN and $s o$-AlN. Overall, the phonon modes of $s o$-AlN are more dispersive compared to that of $s o-\mathrm{GaN}$. For example, $s o-\mathrm{GaN}$ has a gap between 420 and $520 \mathrm{~cm}^{-1}$, while in $s o-A l N$ that region is filled with dispersed bands.

The geometry of the $s o$-structures belongs to the point group $D_{4 h}$. In this respect, the phonon modes are expected to be either nondegenerate or double degenerate at the $\Gamma$ point corresponding to either $A, B$, or $E$ modes, respectively. The phonon modes corresponding to irreducible representations $A_{1 g}, B_{1 g}$, and $B_{2 g}$ are Raman active, while those corresponding to $A_{2 u}$ and $E_{2 u}$ are infrared active.

The character of optical modes at the $\Gamma$ point is revealed for both $s o-\mathrm{GaN}$ and $s o-\mathrm{AlN}$ in Fig. 2(a). The eigenvectors of Raman active modes are presented in Fig. 2(b). All Raman active modes are due to in-plane atomic displacements. Here, the modes are arranged from left to right and from top to bottom in the decreasing order of frequencies. The $B_{2 g}$ mode of $s o-\mathrm{GaN}$ around $420 \mathrm{~cm}^{-1}$ appears around $680 \mathrm{~cm}^{-1}$ in $s o$-AlN. This corresponds to the biggest shift compared to other modes and it is characterized by displacements of $\mathrm{Ga}$ or $\mathrm{Al}$ atoms in opposite directions along the diagonal of the squares. The $A_{2 g}$ mode has similar character and it also has a significant frequency (a)

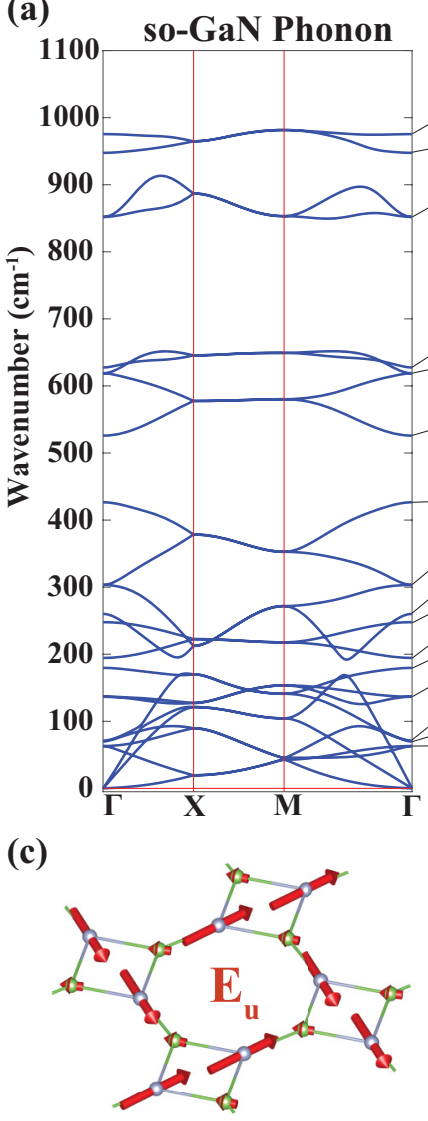

so-AIN Phonon

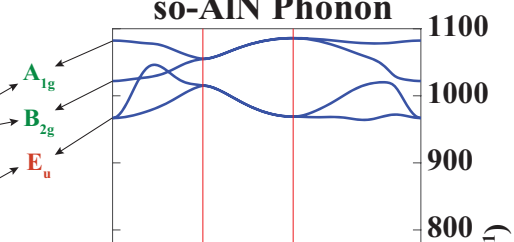

(b)

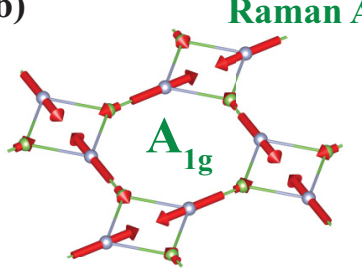

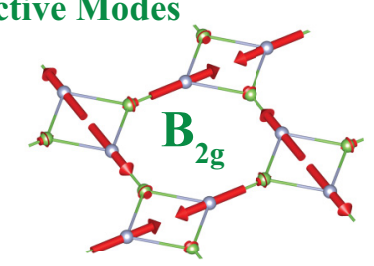
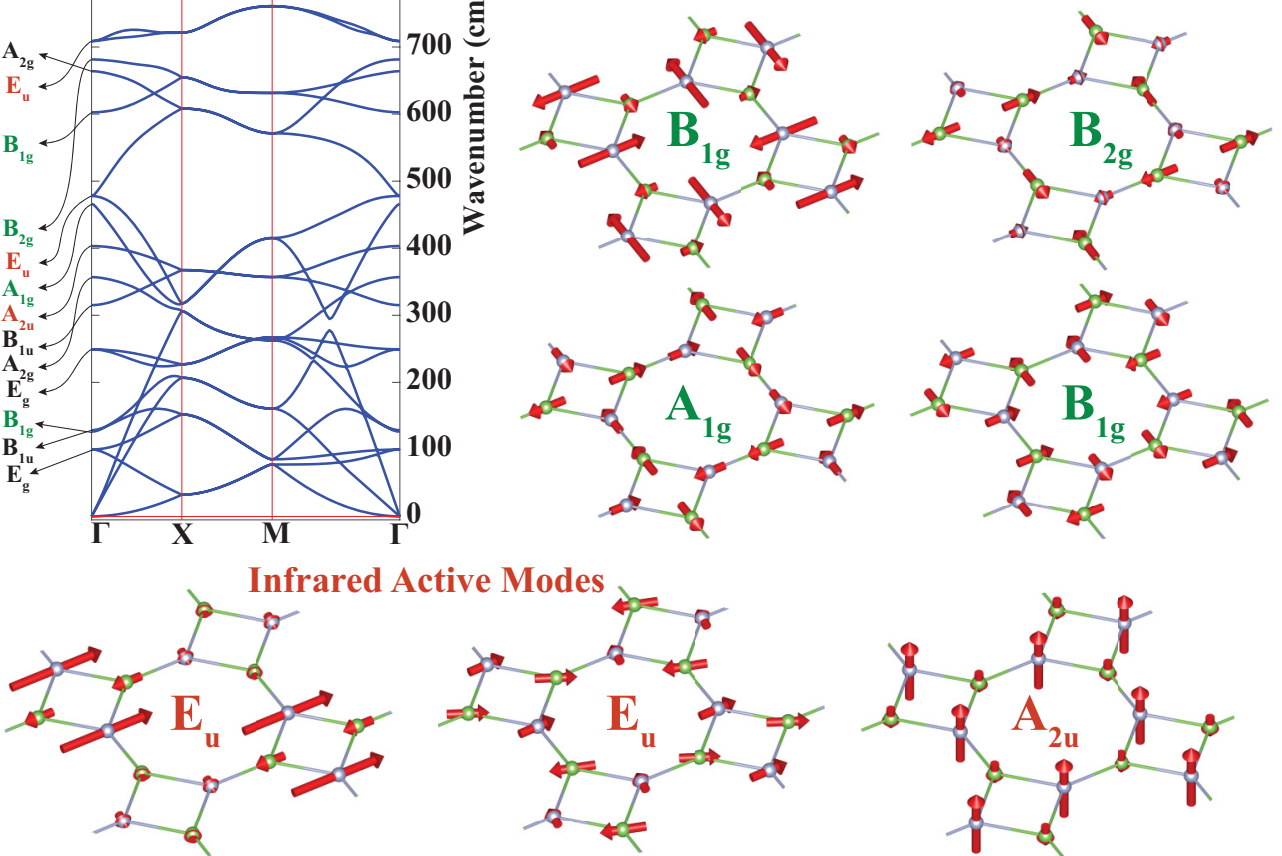

ctive Modes
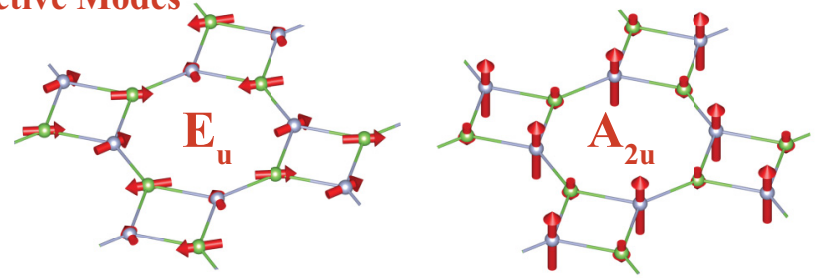

FIG. 2. (a) Phonon dispersions of $s o-G a N$ and so-AlN calculated along major symmetry directions of the Brillouin zone. The symmetries of the modes are indicated. (b) The Raman active modes and (c) the infrared active modes are both arranged in the order of decreasing frequency from left to right and from top to bottom. 

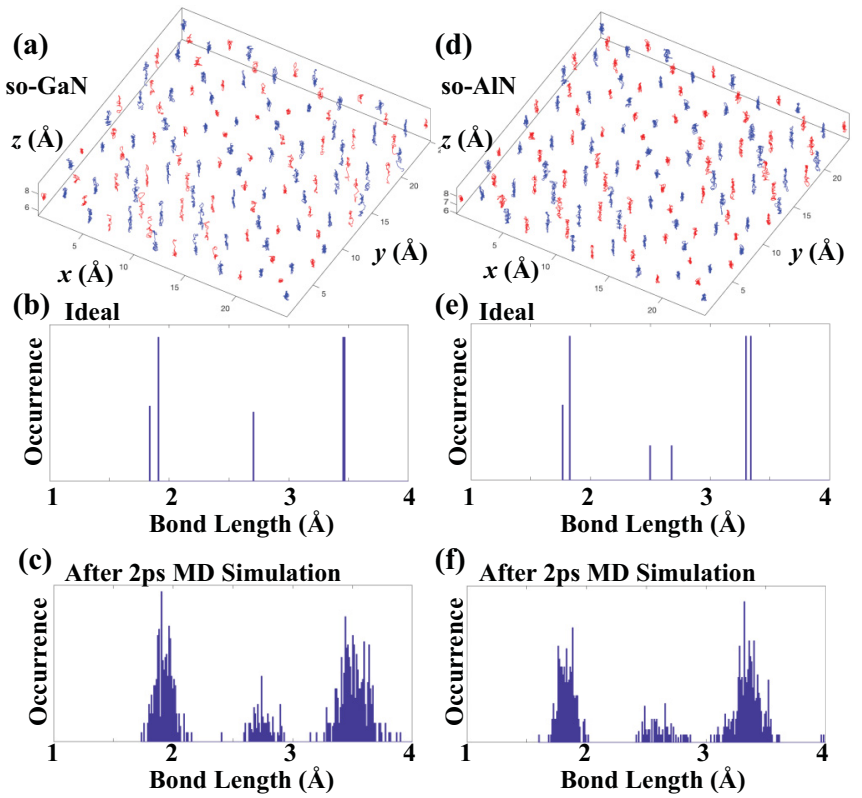

(e) Ideal



(f) After 2ps MD Simulation

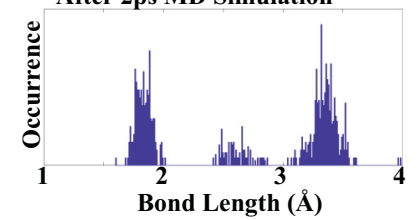

FIG. 3. (a) Trajectories of atoms in $s o-\mathrm{GaN}$ in the course of a 2-ps ab initio MD simulation performed at $1000 \mathrm{~K}$. The units of displacements along $x, y$, and $z$ directions are shown by inset. (b) The bond length occurrence histogram of $s o$-GaN for ideal geometry. (c) Same for the geometry of $s o-\mathrm{GaN}$ at the end of the MD simulation. (d)-(f) Same as (a)-(c) for so-AlN.

shift when two $s o$-structures are compared. The $A_{1 g}$ mode of $s o-\mathrm{GaN}$ around $260 \mathrm{~cm}^{-1}$ has similar character and it also has a significant frequency shift reaching $466 \mathrm{~cm}^{-1}$ in $s o$-AlN.

Three in-plane $E_{u}$ modes and one out-of-plane $A_{2 u}$ mode comprise the infrared active phonons of $s o$-structures. The eigenvectors of these modes are presented in Fig. 2(c) from left to right in the decreasing order of frequencies. The infrared modes are dominated by displacements of $\mathrm{N}$ atoms. In the infrared active modes, two $\mathrm{N}$ atoms in the same square move in the same direction while in the Raman active modes, the opposite is true. The $E_{u}$ branches with the highest frequencies have striking similarity to the Mexican hat dispersion of the valence band edge of so-structures [45]. Hence, the Mexican hat dispersion that appears both in the electronic and phononic band structures can be interpreted as a fundamental property of interactions in the so geometry.

\section{B. Thermal stability}

Even if SL $s o$-structures are dynamically stable at $\mathrm{T}=$ $0 \mathrm{~K}$, their stability against thermal excitation is essential for their stability in possible technological applications. Thermal stability of $s o$-structures was tested at high temperatures by ab initio molecular dynamics (MD) calculations. We simulated microcanonical ensemble using Berendsen thermostat. We use a time step of 2 fs for the integration of the equations of motion. Kinetic energies are rescaled in every 50 steps. We first increase the temperature of the system from 0 to $1000 \mathrm{~K}$ in $2 \mathrm{ps}$ and then keep the system at $1000 \mathrm{~K}$ for another 2 ps. In Figs. 3(a) and 3(d), we present the trajectories of atoms of $s o-\mathrm{GaN}$ and $s o$-AlN in the course of the latter simulation. At temperatures as high as $1000 \mathrm{~K}$, the trajectories are rather local and the displacements of atoms are restored to the equilibrium points. While the occurrence of distances of neighbors marks sharp lines at $T=0 \mathrm{~K}$ in Figs. 3(b) and 3(e), they smear out and overlap with each other after 2-ps MD simulations at $T=1000 \mathrm{~K}$ as shown in Figs. 3(c) and 3(f). Briefly, despite significant displacements of atoms, overall square-octagon structure is maintained and the stability of free-standing $s o$-structures is assured at high temperature.

TABLE I. Calculated values for so-GaN: optimized lattice constant $a$ (for $3 \mathrm{D} w z-\mathrm{GaN} a / c$ ); two different Ga-N bond lengths $d_{1} / d_{2}$; cohesive energy $E_{c}$ per Ga-N pair; in-plane stiffness $C_{1} / C_{2}$, Poisson's ratio $v_{1} / v_{2}$ (see text for definitions); indirect band gap $E_{G-i}$; direct band gap $E_{G-d}$; indirect band gap corrected by HSE, $E_{G \text {-HSE }}$ (direct band gap corrected by HSE is given in parentheses). Corresponding values for hexagonal SL $h-\mathrm{GaN}$ and 3D $w z-\mathrm{GaN}$ are also presented for the sake of comparison. Line marked by $(*)$ comprises values calculated in this study.

\begin{tabular}{|c|c|c|c|c|c|c|c|c|c|c|c|}
\hline & $\left.\begin{array}{c}a \\
(\AA / \AA ̊\end{array}\right)$ & $\begin{array}{c}d_{1} / d_{2} \\
(\AA)\end{array}$ & $\begin{array}{c}E_{c} \\
(\mathrm{eV} / \mathrm{GaN})\end{array}$ & $\begin{array}{c}E_{f} \\
(\mathrm{eV} / \mathrm{GaN})\end{array}$ & $\begin{array}{l}C_{1} / C_{2} \\
(\mathrm{~N} / \mathrm{m})\end{array}$ & $\begin{array}{c}v_{1} / \nu_{2} \\
(\%)\end{array}$ & $\begin{array}{l}E_{G-i} \\
(\mathrm{eV})\end{array}$ & $\begin{array}{l}E_{G-d} \\
(\mathrm{eV})\end{array}$ & $\begin{array}{c}E_{G \text {-HSE }} \\
(\mathrm{eV})\end{array}$ & $\begin{array}{c}E_{G-G W_{0}} \\
(\mathrm{eV})\end{array}$ & $\begin{array}{c}E_{G-G_{0} W_{0}} \\
(\mathrm{eV})\end{array}$ \\
\hline \multicolumn{12}{|l|}{$s o-\mathrm{GaN}$} \\
\hline PBE* & 6.42 & $1.91 / 1.83$ & 7.53 & -1.23 & $41.63 / 125$ & $0.76 / 0.29$ & 1.85 & 2.13 & $3.37(3.69)$ & & \\
\hline PBE [36] & & $1.95 / 1.85$ & 6.76 & & & & 1.60 & & & & \\
\hline PBE [37] & 6.41 & $\sim 1.83$ & & -0.12 & & & 1.89 & & 3.12 & & \\
\hline \multicolumn{12}{|l|}{$w z-\mathrm{GaN}$} \\
\hline PBE [21] & $3.22 / 5.24$ & 1.97 & 8.76 & & 171 & 0.18 & & 1.71 & $3.48(2.96)$ & & \\
\hline \multicolumn{12}{|l|}{$h-\mathrm{GaN}$} \\
\hline PBE [21] & 3.21 & 1.85 & 8.29 & & 109.8 & 0.43 & 2.16 & & 3.42 & & 4.55 \\
\hline LDA [6] & 3.20 & 1.85 & 12.74 & & 110 & 0.48 & 2.27 & & & 5.0 & \\
\hline PBE [47] & 3.21 & 1.85 & & & 109.4 & 0.43 & & & & & \\
\hline PBE [48] & & 1.85 & 8.38 & & & & 2.17 & & & & \\
\hline PBE [18] & & 1.87 & 8.06 & & & & 1.87 & & & 4.14 & \\
\hline LDA [49] & 3.17 & & & & & & 2.36 & & & & 4.27 \\
\hline PBE [25] & 3.25 & & & & & & & & 3.23 & & 4.00 \\
\hline
\end{tabular}




\section{Mechanical properties}

Further to dynamical and thermal stability analysis, we calculate the mechanical properties of $\mathrm{SL}$ so-structures including the in-plane stiffness and the Poisson's ratio [46]. Mechanical properties of both structures are investigated in the harmonic range of the elastic deformation, where the structure responded to strain, $\epsilon$ linearly. We first apply strain varying between -0.01 and +0.01 along the lattice vectors that lie on $x$ and $y$ axes and calculate the total energy $E_{T}\left[\epsilon_{x}, \epsilon_{y}\right]$ for each case. Here, $\epsilon_{x}$ and $\epsilon_{y}$ are applied strains along $x$ and $y$ axes, respectively. Then, we fit the results to the following quadratic polynomial:

$$
E_{T}\left[\epsilon_{x}, \epsilon_{y}\right]-E_{T}\left[\epsilon_{x}=0, \epsilon_{y}=0\right]=a_{1} \epsilon_{x}^{2}+a_{2} \epsilon_{y}^{2}+a_{3} \epsilon_{x} \epsilon_{y},
$$

where $a_{1}, a_{2}$, and $a_{3}$ are fitting parameters and $E_{T}\left[\epsilon_{x}=0\right.$, $\left.\epsilon_{y}=0\right]$ is the energy of the unstrained system. Here, $a_{1}=a_{2}$ because of the symmetry. The in-plane stiffness $\left(C_{1}\right)$ and the Poisson's ratio $\left(v_{1}\right)$ corresponding to the uniaxial strain along the lattice vectors can be expressed in the following way:

$$
\begin{aligned}
v_{1} & =\frac{a_{3}}{2 a_{1}}, \\
C_{1} & =\frac{4 a_{1}^{2}-a_{3}^{2}}{2 a_{1} A_{0}},
\end{aligned}
$$

where $A_{0}$ is the area of the unstrained unit cell.

We also calculated the in-plane stiffness and the Poisson's ratio corresponding to the uniaxial strain along the diagonals of the unit cell that lie on $x=y$ and $-y$ lines. In this case, we apply strain ranging from -0.01 to 0.01 along $y=x$ and $-x$ lines and calculate the total energy $E_{T}\left[\epsilon_{y=x}, \epsilon_{y=-x}\right]$. Here, $\epsilon_{y=x}$ and $\epsilon_{y=-x}$ are applied strains along $y=x$ and $-x$ lines, respectively. Then, we fit the results to the following quadratic polynomial:

$$
\begin{gathered}
{[b] E_{T}\left[\epsilon_{y=x}, \epsilon_{y=-x}\right]-E_{T}\left[\epsilon_{y=x}=0, \epsilon_{y=-x}=0\right]} \\
=b_{1} \epsilon_{y=x}^{2}+b_{2} \epsilon_{y=-x}^{2}+b_{3} \epsilon_{y=x} \epsilon_{y=-x},
\end{gathered}
$$

where $b_{1}, b_{2}$, and $b_{3}$ are fitting parameters. The in-plane stiffness $\left(C_{2}\right)$ and the Poisson's ratio $\left(v_{2}\right)$ corresponding to the uniaxial strain along the diagonals of the unit cell can be expressed in the following way:

$$
\begin{aligned}
v_{2} & =\frac{b_{3}}{2 b_{1}}, \\
C_{2} & =\frac{4 b_{1}^{2}-b_{3}^{2}}{2 b_{1} A_{0}} .
\end{aligned}
$$

Calculated values for $C_{1}, C_{2}, v_{1}$, and $v_{2}$ are listed in Table I. Notice that $C_{2}$ values are about three times higher than $C_{1}$ values, which means that the $s o$-structures are stiffer against the strain applied along the diagonals compared to the one applied along the lattice vectors. Concomitantly, $v_{1}$ values are about three times higher than $v_{2}$ values.

\section{ELECTRONIC STRUCTURE}

The electronic energy band structure of SL so-GaN and so-AlN calculated using PBE are presented in Fig. 4. Both structures are wide, indirect-band-gap semiconductors. In Tables I and II, relevant values concerning the electronic structure are presented. Those values of SL $h-\mathrm{GaN}, h$-AlN, 3D $w z-\mathrm{GaN}$, and 3D $w z-\mathrm{AlN}$ are also included for comparison. In Fig. 4(a), the indirect band gap of $s o-\mathrm{GaN}$ is $E_{g, i}=1.85 \mathrm{eV}$ and occurs between the minimum of conduction band (CB) at the $\Gamma$ point and maximum of the valence band (VB) along $\Gamma-X$ direction. The lowest conduction band is constructed from antibonding $s$ orbitals with small $p_{x y}$ contribution. The total density of states (TDOS) is low at the edge of CB due to the high dispersion of this band. The highest valence bands have

\begin{tabular}{|c|c|c|c|c|c|c|c|c|c|c|c|}
\hline & $(\stackrel{a}{\AA} / \AA)$ & $\begin{array}{c}d \\
(\AA)\end{array}$ & $\begin{array}{c}E_{c} \\
(\mathrm{eV} / \mathrm{AlN})\end{array}$ & $\begin{array}{c}E_{f} \\
(\mathrm{eV} / \mathrm{AlN})\end{array}$ & $\begin{array}{l}C_{1} / C_{2} \\
(\mathrm{~N} / \mathrm{m})\end{array}$ & $\begin{array}{l}v_{1} / v_{2} \\
(\%)\end{array}$ & $\begin{array}{l}E_{G-i} \\
(\mathrm{eV})\end{array}$ & $\begin{array}{l}E_{G-d} \\
(\mathrm{eV})\end{array}$ & $\begin{array}{c}E_{G-\mathrm{HSE}} \\
(\mathrm{eV})\end{array}$ & $\begin{array}{c}E_{G-G W_{0}} \\
(\mathrm{eV})\end{array}$ & $\begin{array}{c}E_{G-G_{0} W_{0}} \\
(\mathrm{eV})\end{array}$ \\
\hline \multicolumn{12}{|l|}{ so-AlN } \\
\hline PBE* & 6.15 & $1.83 / 1.76$ & 10.05 & -2.23 & $38.22 / 143.67$ & $0.79 / 0.22$ & 2.87 & 3.03 & $4.09(4.26)$ & & \\
\hline PBE [38] & & $1.83 / 1.76$ & 10.18 & & & & 2.86 & & & & \\
\hline \multicolumn{12}{|l|}{$w z-\mathrm{AlN}$} \\
\hline PBE [20] & $3.11 / 5.01$ & 1.90 & 12.28 & & & & & 4.21 & & & \\
\hline \multicolumn{12}{|l|}{$h$-AlN } \\
\hline PBE [21] & 3.13 & 1.81 & 10.56 & & 114 & 0.46 & 2.91 & 3.62 & 4.00 & & \\
\hline LDA [6] & 3.09 & 1.79 & 14.30 & & 116 & 0.46 & 3.08 & & & 5.57 & \\
\hline PBE [20] & 3.13 & 1.81 & 10.72 & & & & 2.92 & 3.62 & 4.06 & & \\
\hline PBE [50] & 3.17 & 1.83 & 10.01 & & & & 2.88 & & & & \\
\hline PBE [25] & 3.13 & & & & & & & & 4.85 & & 5.03 \\
\hline PBE [51] & 3.13 & & & & & & 2.91 & & & & \\
\hline$G_{0} W_{0}[52]$ & 3.03 & & & & & & & & & & \\
\hline
\end{tabular}
low dispersion; one of them has maximum away from the

TABLE II. Calculated values for so-AlN: optimized lattice constant $a$ for $3 \mathrm{D} w z$-AlN $a / c)$; two different Al-N bond lengths $d_{1} / d_{2}$; cohesive energy $E_{c}$ per Al-N pair; in-plane stiffness $C_{1} / C_{2}$, Poisson's ratio $v_{1} / v_{2}$ (see text for definitions); indirect band gap $E_{G-i}$; direct band gap $E_{G-d}$; indirect band gap corrected by HSE, $E_{G \text {-HSE }}$ (direct band gap corrected by HSE is given in parentheses). Corresponding values for hexagonal SL $h$-AlN, SL $t$-AlN and 3D $w z$-AlN are also presented for the sake of comparison. Line marked by $(*)$ comprises values calculated in this study. 



FIG. 4. (a) Electronic energy band structure of $s o-\mathrm{GaN}$ and the densities of states projected on constituent $\mathrm{Ga}$ and $\mathrm{N}$ atoms. PBE indirect band gap $E_{G, i}$ is shown by arrow. Bands corrected by HSE are shown by dashed lines. HSE indirect band gap becomes $E_{G, i}=$ $3.37 \mathrm{eV}$ after HSE correction. Dominant orbital character of the bands are indicated. (b) Same as (a) for so-AlN. After HSE correction, the indirect band gap becomes $E_{g, i}=4.09 \mathrm{eV}$. (c) Isosurface charge density of of states at the valence band and conduction band edges. (d) Same as (c) for so-AlN.

center of the BZ. This corresponds to the so-called "Mexican hat" dispersion. Accordingly, TDOS near the edge of VB is high. These two bands are derived from $p_{z}$ orbitals of nitrogen atoms. The energy bands of so-AlN presented in Fig. 4(b) as well as corresponding TDOS display features similar to those of $s o-\mathrm{GaN}$, except the indirect band gap which is relatively larger and $E_{g, i}=2.78 \mathrm{eV}$. In both SL structures states projected to $\mathrm{N}$ atoms dominate TDOS of VB for $-3 \mathrm{eV}<E<0 \mathrm{eV}$. Charge density isosurfaces of the bands at the edge of $\mathrm{CB}$ and $\mathrm{VB}$ of $s o-\mathrm{GaN}$ and $s o-\mathrm{AlN}$ presented in Figs. 4(c) and 4(d) confirm the above arguments about the character of these bands. These band gaps are smaller than the indirect band gaps of SL $h$-GaN and $h$-AlN. While the fundamental band gap of bulk 3D $w z-\mathrm{GaN}$ calculated using $\mathrm{PBE}$ is direct and smaller than the corresponding indirect band gaps of SL so-GaN, the direct fundamental band gap of 3D $w z$-AlN calculated PBE is larger than the indirect band gap of SL so-AlN. This order is unaltered even after HSE corrections. The same paradoxical trends exist between 3D $w z$-structures and $h$-structures. Namely, while the fundamental band gap of bulk 3D wz-AlN calculated using PBE is direct and smaller than the corresponding indirect band gaps of SL $h-\mathrm{GaN}$, the direct fundamental band gap of 3D $w z$-AlN calculated PBE is larger than the indirect band gap of SL $h$-AlN. The increase of the fundamental band gap of GaN by going from 3D to SL 2D can be reconciled with the confinement effect; we think that another effect related with the polarity of bonds in AlN might reduce the effect of the confinement by going from $3 \mathrm{D}$ to $2 \mathrm{D}$, and hence be responsible for the above paradoxical situation.

Since PBE underestimates the fundamental band gap, the PBE bands are corrected by using HSE [53]. The indirect band gaps of $s o-\mathrm{GaN}$ and $s o$-AlN increased upon HSE correction to $E_{g, i}=3.37$ and $=4.09 \mathrm{eV}$, respectively. The calculated electronic properties confirm that SL so-structures are stable wide-band semiconductors promising potential applications similar to SL hexagonal structures and 3D wz-bulk crystals.

\section{A. Effect of strain}

Uniaxial or biaxial strain are known to provide a tool to tune the fundamental band gap of 2D SL and multilayer structures. The effects of uniaxial and biaxial strains on the fundamental band gap are presented in Fig. 5. The uniaxial strain $\epsilon_{x}$ is applied along the $x$ direction and the structure is optimized by keeping the lattice constant $a_{1}^{\prime}=a_{1}\left(1+\epsilon_{x}\right)$ fixed, but relaxing $a_{2}^{\prime}$ and all the atomic positions to minimize the total energy of the strained system. For the optimized structure corresponding to each applied value of $\epsilon_{x}$ the electronic band structure is calculated and the fundamental band gap is determined. In the biaxial case, the structure is optimized with fixed lattice constant $a_{1}^{\prime}=a_{2}^{\prime}=a_{1}(1+\epsilon)$. The fundamental band gap of each optimized structure is calculated to plot $E_{g, i}$ versus strain.

In Fig. 5(a), the fundamental band gap of SL so-GaN decreases with increasing uniaxial strain $\epsilon_{x}$. In the biaxial strain case $\left(\epsilon_{x}=\epsilon_{y}\right)$, the variation of band gap is almost linear with $\epsilon$; the gap increases with compression, but decreases with

TABLE III. Optimized total energies ( $E_{T}$ per unit cell), the length of vertical $C$ - $A$ bonds $\left(d_{v}\right)$, and buckling $(\Delta)$ calculated for bilayers having different stacking geometries $\left(A A^{\prime}, A B\right.$, and cubane) using different vdW corrections.

\begin{tabular}{|c|c|c|c|c|c|c|c|c|c|c|}
\hline & & \multicolumn{3}{|c|}{$A A^{\prime}$} & \multicolumn{3}{|c|}{$A B$} & \multicolumn{3}{|c|}{ Cubane } \\
\hline & & $E_{T}(\mathrm{eV})$ & $d_{v}(\AA)$ & $\Delta(\AA)$ & $\overline{E_{T}(\mathrm{eV})}$ & $d_{v}(\AA)$ & $\Delta(\AA)$ & $E_{T}(\mathrm{eV})$ & $d_{v}(\AA)$ & $\Delta(\AA)$ \\
\hline \multirow{4}{*}{$\begin{array}{l}Z \\
\text { Zू } \\
\vdots \\
\vdots \\
\infty\end{array}$} & vdW-1 [54] & -90.91 & 2.24 & 0.07 & -90.54 & 2.11 & 0.65 & -90.88 & 2.09 & $0.62 / 0.84$ \\
\hline & $\mathrm{vdW}-2$ [55] & -91.84 & 2.23 & 0.09 & -91.41 & 2.11 & 0.65 & -91.73 & 2.09 & $0.61 / 0.83$ \\
\hline & vdW-3 [56] & -92.22 & 2.24 & 0.07 & -92.07 & 2.13 & 0.63 & -92.37 & 2.10 & $0.58 / 0.79$ \\
\hline & $\mathrm{vdW}-4$ [57] & -91.51 & 2.23 & 0.09 & -91.32 & 2.11 & 0.66 & -91.58 & 2.09 & $0.64 / 0.86$ \\
\hline \multirow{4}{*}{$\underset{\substack{Z \\
0 \\
0}}{Z}$} & vdW-1 [54] & -112.89 & 2.11 & 0.12 & -111.30 & 2.03 & 0.54 & & & \\
\hline & $\mathrm{vdW}-2$ [55] & -114.33 & 2.10 & 0.12 & -112.42 & 2.03 & 0.53 & & & \\
\hline & vdW-3 [56] & -113.76 & 2.11 & 0.12 & -112.31 & 2.04 & 0.53 & & & \\
\hline & vdW-4 [57] & -113.66 & 2.11 & 0.12 & -112.27 & 2.03 & 0.54 & & & \\
\hline
\end{tabular}


(a) Uniaxial Strain so-GaN

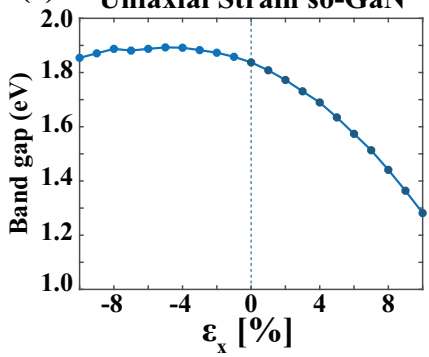

(c) Uniaxial Strain so-AIN

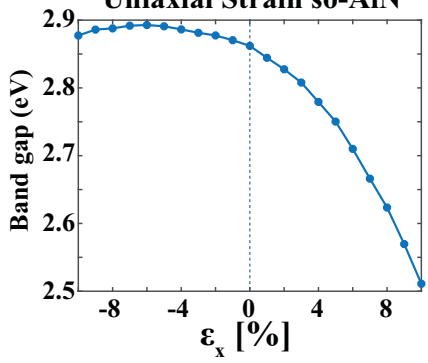

(b) Biaxial Strain so-GaN

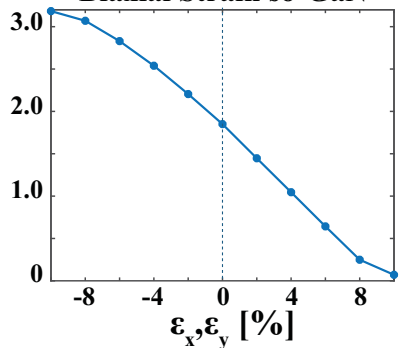

(d) Biaxial Strain so-AIN



FIG. 5. Effects of uniaxial and biaxial strain on the fundamental band gap. (a) Fundamental band gap of SL $s o-G a N$ versus applied uniaxial strain $\epsilon_{x}$. (b) Fundamental band gap of SL $s o$-GaN versus biaxial strain $\epsilon_{x}=\epsilon_{y}$. (c) and (d) Same as (a) and (b), respectively, for SL so-AlN.

expansion. For $\epsilon_{x}=\epsilon_{y}>0.1$, the gap is closed. In the case of SL $s o$-AlN presented in Fig. 5(c), the fundamental band gap decreases with increasing uniaxial strain. Under biaxial strain, SL so-AlN exhibits a behavior similar to that shown by so-GaN.

\section{B. Bilayers and multilayers}

The atomic and electronic structures of SL structures of $\mathrm{GaN}$ and $\mathrm{AlN}$ are subject to changes upon BL and ML formation. Perpendicular cation-anion bonds can form between single layers for specific stacking geometries of BL and result in the buckling in the planar geometry. These structural transformations involving interlayer chemical interactions in addition to van der Waals ( $\mathrm{vdW}$ ) attraction give rise to marked changes in the electronic structure. Here, stacking geometry, dominant interlayer interactions, as well as number of layers in ML formation become crucial parameters to control the properties of these artificial materials. The perpendicular cation-anion bonds are not as strong as lateral bonds and display a different character, but they provide interlayer interaction stronger than vdW interaction and they become more influential on the physical properties.

Here, we first carried out systematic calculations by using different vdW corrections [54-57] included to GGA within PBE (see Table III) for the optimized interlayer distances of well-known layered materials like graphite and layered $\mathrm{MoS}_{2}$, etc. Based on the comparison between predicted and experimental interlayer distances we choose the vdW correction due to Grimme et al. [55] in all bilayer and multilayer calculations in this study.

(a)

Bilayer so-GaN Cubane
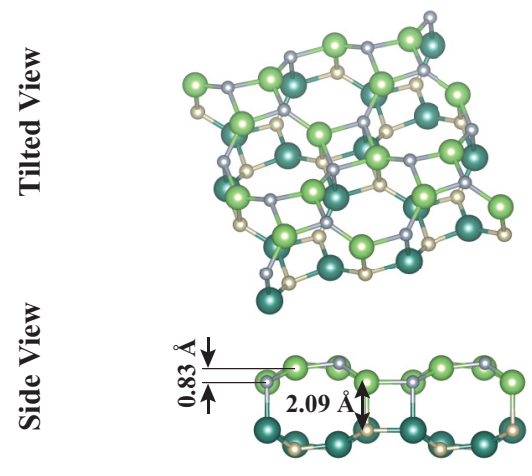

(b)

Bilayer so-GaN AB Bilayer so-AIN AB
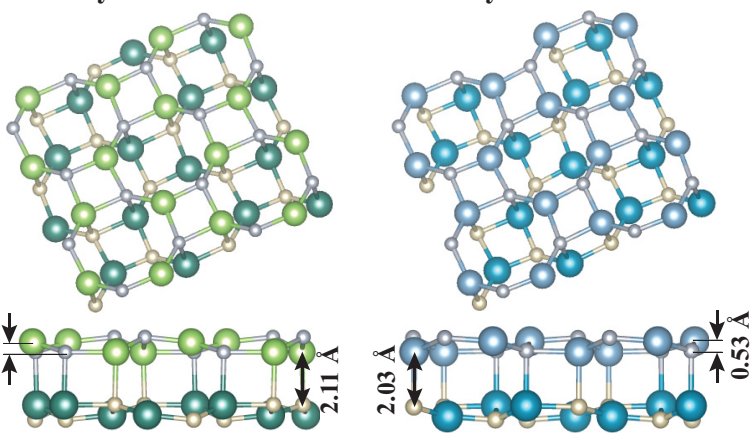

(c)
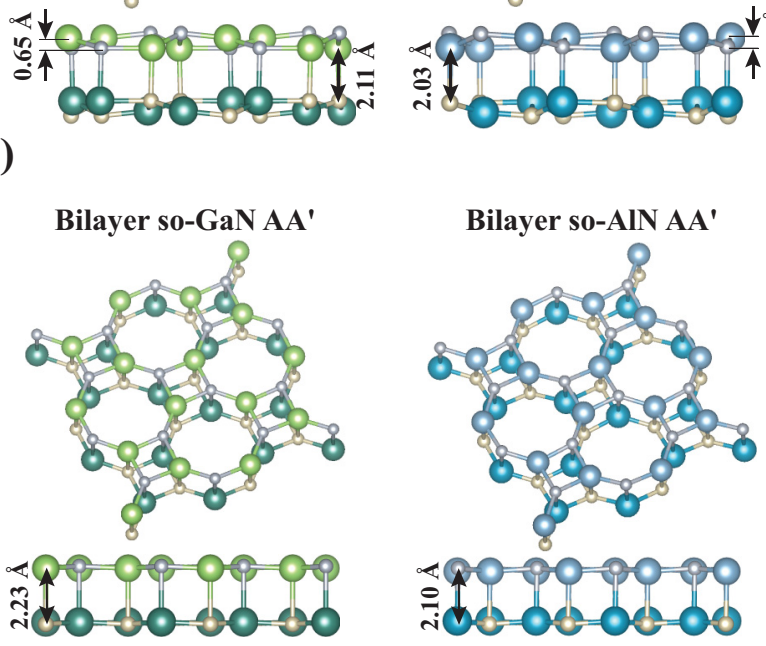

(d)
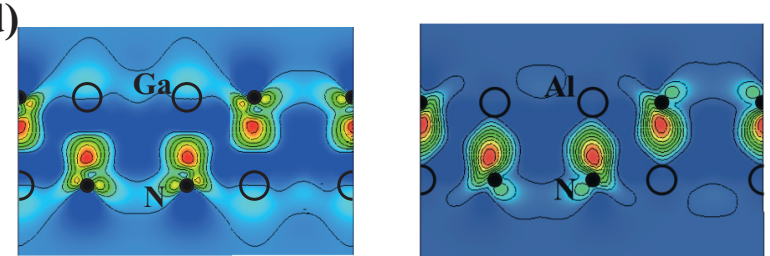

FIG. 6. (a) Tilted and side views of cubane structure of $s o-G a N$ to form a bilayer (BL). (b) Tilted and side views of BL constructed by $A B$ stacking. (c) Tilted and side views of BL constructed from $A A^{\prime}$ stacking. The lengths of vertical cation-anion bonds and the buckling of cation and anion in the same layer are indicated. Cations Ga and $\mathrm{Al}$ are shown by large green and large blue balls, respectively. Anion $\mathrm{N}$ is shown by small gray balls. (d) Charge density difference, $\Delta \rho(r)$, contour plot of BL is obtained by subtracting the charge densities of two SL $s o$-structures from that of BL. Vertical bonds constructed from $\mathrm{Ga} p_{z}-\mathrm{N} p_{z}$ orbitals are clearly seen. Charge density increases from dark blue-light blue-green-yellow-red via color code. 
TABLE IV. Energetics, average layer binding energy, and fundamental band gaps of BL, TL, and 3D layered structures of so-GaN and $s o$-AlN formed by different stacking of corresponding planar SLs. Columns are stacking geometry; $E_{T}$ total energy (eV per in-plane $C$ - $A$ pair); $E_{l b}$ layer binding energy ( $\mathrm{V}$ per layer per unit cell); fundamental band gap (eV) with $d$ direct or $i$ indirect. For the sake of comparison, the total energy of SL $s o-\mathrm{GaN}(s o-\mathrm{AlN})$ is $-11.08 \mathrm{eV}(-13.63 \mathrm{eV})$ per $C-A$ pair. The total energy of $3 \mathrm{D} w z$ structure of GaN $(\mathrm{AlN})$ is $-12.65 \mathrm{eV}$ $(-15.43 \mathrm{eV})$ per $C$ - $A$ pair. All total energies include vdw correction [55].

\begin{tabular}{|c|c|c|c|c|c|c|c|c|c|c|}
\hline & \multirow[b]{2}{*}{ Stacking } & \multicolumn{3}{|c|}{ Bilayer } & \multicolumn{3}{|c|}{ Trilayer } & \multicolumn{3}{|c|}{ Bulk } \\
\hline & & $E_{T}$ & $E_{l b}$ & $E_{g}$ & $E_{T}$ & $E_{l b}$ & $E_{g}$ & $E_{T}$ & $E_{l b}$ & $E_{g}$ \\
\hline \multirow{3}{*}{ 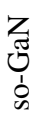 } & $A A^{\prime}$ & -11.48 & 1.60 & $1.58(i)$ & -11.59 & 2.05 & $1.53(i)$ & -11.88 & 3.20 & $1.27(d)$ \\
\hline & $\mathrm{AB}$ & -11.43 & 1.39 & $1.52(i)$ & -11.36 & 1.11 & $1.11(i)$ & -12.39 & 5.24 & $1.60(d)$ \\
\hline & Cubane & -11.47 & 1.55 & $1.48(d)$ & -11.67 & 2.35 & $1.36(d)$ & -12.09 & 4.03 & $1.59(d)$ \\
\hline Z & $A A^{\prime}$ & -14.25 & 2.48 & $3.06(i)$ & -14.44 & 3.23 & $2.93(i)$ & -14.92 & 5.14 & $2.24(d)$ \\
\hline 飞 & $\mathrm{AB}$ & -14.05 & 1.67 & $2.35(i)$ & -14.03 & 1.56 & $2.20(i)$ & -15.22 & 6.33 & $3.66(d)$ \\
\hline o & Cubane & & & & -14.37 & 2.95 & $2.46(d)$ & -14.89 & 5.04 & $3.04(d)$ \\
\hline
\end{tabular}

As shown in Figs. 6(a)-6(c), among all other possible geometries, three stacking geometries in BL are critical: these are cubane, $A B$, and $A A^{\prime}$. In cubane-type stacking, the primitive unit cells of two layers are exactly on top of each other, except the positions of cations (anions) in the bottom layer are exchanged by anions (cations) in the adjacent layer. Additionally, four vertical cation-anion bonds are pressed to form a cubane of $\mathrm{Ga}-\mathrm{N}$ bonds, whereas other vertical bonds remained relatively longer. This way, single layers forming this structure acquired a significant buckling and attained haeckelite form even at side view. Interestingly, a similar situation is found in the multilayer structures of $h$-GaN [23]. However, cubane BL of so-AlN did not form; the structure starting from cubane $\mathrm{BL}$ transforms to $A A^{\prime} \mathrm{BL}$ in the course of structure optimizations. Apparently, cubane BL of so-AlN does not have a local minimum. In the $A B$ stacking, the centers of squares of the top layer are located at the centers of bottom octagons. This way, BL has four vertical bonds of $2.11 \AA$ and has significant buckling. In $A A^{\prime}$ stacking, the primitive unit cells of two layers are exactly on top of each other, except the positions of cations (anions) in the bottom layer are exchanged by anions (cations) in the adjacent layer and layers remained planar with negligible buckling. Eventually, eight vertical cation-anion bonds can form between two layers. This way, each cation in the bilayer is fourfold coordinated by anions and vice versa; among these four cation-anion bonds one bond, that is, the vertical bond, is $\sim 17 \%$ larger than three other in-plane $d_{1} / d_{2}$ bonds. Namely, while the lengths of in-plane Ga-N bonds are 1.83-1.91 $\AA$, the length of vertical Ga-N bonds is $2.23 \AA$. The charge density difference calculated by subtracting the charge densities of the top and bottom SL layers from the charge density of BL, i.e., $\Delta \rho(r)=\rho_{\mathrm{BL}}(r)-\rho_{\mathrm{SL}_{1}}(r)-\rho_{\mathrm{SL}_{2}}(r)$ shown in Fig. 6(d), clearly indicates vertical covalent+ionic bonding between cation and anion. Similar stacking geometries and sequences followed also for trilayers (TL) and periodic 3D layered structures. Calculated total energies, layer binding energies, and band gaps of $\mathrm{BL}, \mathrm{TL}$, and 3D layered structures are presented in Table IV.

Among three stacking geometries, BL with $A A^{\prime}$ stacking has lowest total energy. Relatively longer eight vertical bonds in the unit cell imply weaker chemical interaction. The binding energy between two $s o$-GaN layers of $\mathrm{BL}$ in $A A^{\prime}$ stacking comprises vdW and chemical interaction and is calculated to be $400 \mathrm{meV}$ per vertical $\mathrm{Ga}-\mathrm{N}$ bond (or $1.60 \mathrm{eV}$ per layer per unit cell). This is stronger than the usual vdW interaction and includes a significant contribution from weak $\mathrm{Ga}-\mathrm{N}$ vertical bonds constructed on $\mathrm{Ga} p_{z}-\mathrm{N} p_{z}$ orbitals. Owing to the formation of vertical bonds, planar $s o-\mathrm{GaN}$ is slightly buckled by $\Delta=0.09 \AA$.

A similar situation arises in TL structure of $A A^{\prime}$ stacking with slightly longer bond lengths and slightly lower binding energy of layers with $382 \mathrm{meV}$ per vertical Ga-N bond. In 3D periodic layered structure of $A A^{\prime}$ stacking, the length of the vertical bond is $2.25 \AA$ and the binding energy is $399 \mathrm{meV}$ per vertical bond. BL, TL, and $3 \mathrm{D}$ periodic layered structure of so-AlN in $A A^{\prime}$ stacking sequence displays similar trend with layer binding energies 620,607, and $645 \mathrm{meV}$ per vertical Al-N bonds. 3D periodic layered structures of $s o-\mathrm{GaN}$ and so-AlN starting from the $A B$ stacking underwent significant relaxation in the course of optimization and transformed to structures similar to 3D $w z-\mathrm{GaN}$ and $w z$-AlN. These structures are most energetic (have lowest total energy) among 3D layered structures having $A A^{\prime}$ and cubane stacking, but they are still less energetic relative to $3 \mathrm{D} w z$-structures in the global minimum.

In closing this part, we emphasize that the interlayer interaction in multilayers of so-GaN and so-AlN involves chemical interaction in addition to attractive $\mathrm{vdW}$, and hence it is usually stronger than that in van der Waals solids. Based on ab initio phonon and finite-temperature MD calculations we found that multilayers and 3D layered structures are stable. Apparently, multilayers in Table IV constitute a different class of vertically layered structures, which is intermediate between $\mathrm{vdW}$ and wurtzite/zinc-blende crystals.

\section{CHEMICAL DOPING OF SELECTED ADATOMS}

Interaction between $s o$-structures and single foreign atoms is important. Mechanical, electronic, and magnetic properties can be modified by foreign atoms. In this paper, we studied the chemisorption of single $\mathrm{H}, \mathrm{O}, \mathrm{N}, \mathrm{Ga}$ atoms on SL so-GaN and $\mathrm{H}, \mathrm{O}, \mathrm{N}$, and $\mathrm{Al}$ atoms on SL so-AlN. To mimic the adsorption of single (isolated) atom to SL $s o$-structures we considered one adatom adsorbed at the same position of $(4 \times 4)$ supercell with $(3 \times 3 \times 1) k$-point sampling. A supercell of this 



FIG. 7. Projected densities of states (PDOS) of $\mathrm{H}$ and $\mathrm{O}$ adatoms adsorbed to SL $s o-\mathrm{GaN}$ and so-AlN. Positions of adatoms are highlighted by cross signs. The zero of energy is set to the common Fermi level of adatom+so-substrate. The band gap of the bare $s o$-structure is shaded. Analysis of PDOSs reveal localized states originating from adatom adsorption. Isosurfaces of the charge densities of the specific states forming marked peaks in the densities of states are also presented.

size is sufficient to minimize the adatom-adatom coupling. To find the equilibrium position of an adatom on the SL $s o$-structures, we calculated total energy for various adsorption sites and minimized it by varying its height. The adsorption site corresponding to the lowest total energy is taken as the equilibrium site for a given atom. The binding energy is calculated as $E_{b}=E_{T}$ [bare $\left.s o\right]+E_{T}[A]-E_{T}[A+s o]$ in terms of the total energies of bare SL $s o$-structure, of free adatom and of adatom adsorbed to $s o$-structure, respectively. $E_{b}>0$ indicates adatom's binding is favored energetically. In Table $\mathrm{V}$, the optimized equilibrium sites of adatoms, their binding energies, and magnetic moments are given.

TABLE V. Selected adatoms adsorbed on $s o-G a N$ and $s o-A l N$; equilibrium configuration or adsorption site; binding energy $E_{b}$; magnetic moment $\mu$. TN $=$ top of $\mathrm{N}$ atom; $\mathrm{N}_{2}$ molecule embedded in the layer; $\mathrm{D}=$ dumbbell structure.

\begin{tabular}{ccccc}
\hline \hline & & Configuration & $E_{b}(\mathrm{eV})$ & $m\left(\mu_{s}\right)$ \\
\hline & $\mathrm{H}$ & $\mathrm{TN}$ & 2.05 & 0 \\
$Z$ & $\mathrm{O}$ & $\mathrm{TN}$ & 3.15 & 0 \\
0 & $\mathrm{~N}$ & $N_{2}$ & 2.66 & 1.0 \\
$\vdots$ & $\mathrm{D}$ & 1.69 & 1.0 \\
$\vdots$ & $\mathrm{Ga}$ & $\mathrm{D}$ & 1.27 & 1.0 \\
& $\mathrm{H}$ & $\mathrm{TN}$ & 3.83 & 0 \\
& $\mathrm{O}$ & $\mathrm{TN}$ & 2.77 & 1.0 \\
$Z$ & $\mathrm{~N}$ & $\mathrm{TN}$ & 1.27 & 1.0 \\
\hline & $\mathrm{TN}$ & $\mathrm{TN}$ & & \\
$\vdots$ & $\mathrm{Al}$ &
\end{tabular}

All adatoms treated in this study form strong chemisorption bonds with SL so-substrates with binding energies ranging from 1.27 to $3.83 \mathrm{eV}$. $\mathrm{H}$ and $\mathrm{O}$ atoms are adsorbed on top of $\mathrm{N}$ atoms of the $s o$-substrates. $\mathrm{N}$ adatom favors to form $\mathrm{N}_{2}$ formation, which is embedded to the plane of $s o-\mathrm{GaN}$ forming bonds with nearby atoms. Ga adatom forms a dumbbell structure on $\mathrm{SL}$ so-GaN by pushing the host Ga down from its plane.

The effects of the adatoms $\mathrm{H}$ and $\mathrm{O}$ on the electronic structure of SL so-structures are analyzed in Fig. 7 in terms of total (TDOS) and atom projected (PDOS) densities of states. To determine the energy positions of the adatom driven localized states relative to the band edges of the bare $s o$-structure, we also calculated the density of states projected on a cation-anion pair far from the adatom in the supercell [58]. The projected density of states calculated this way sets the fundamental band gap of the large, bare $s o$-structures. In the same figure, we also present charge density isosurfaces of specific states corresponding to the peaks in the adatom projected density of states.

Hydrogen adatom adsorbed on top of $\mathrm{N}$ atom gives rise to the $2 p_{z}$-like states near the conduction band edge, which are localized at second-nearest-neighbor $\mathrm{N}$ atoms of $s o-\mathrm{GaN}$. In the case of $s o-\mathrm{AlN}$, states localized at $\mathrm{N}$ atom just below $\mathrm{H}$ occur below and above the Fermi level. Oxygen adatom adsorbed on top of $\mathrm{N}$ atom gives rise to localized states below the valence band edge and above the conduction band edge of $s o-G a N$. States of $\mathrm{O}$ adatom adsorbed on top of $\mathrm{N}$ atom of $\mathrm{so}$ AlN occur at the edge of the conduction band. Permanent and local magnetic moments of 1.0 bohrs magneton are attained upon the adsorption of $\mathrm{N}$ and $\mathrm{Ga}$ adatoms to SL $s o-\mathrm{GaN}$ and upon the adsorption of $\mathrm{H}, \mathrm{N}$, and $\mathrm{Al}$ adatoms to SL so-AlN. Adatom induced localized states and their charge distribution can be imaged by STM [59].

\section{VACANCY FORMATION}

A vacancy of cation-anion pair formed in a so-structure gives rise to localized states, which can influence the electronic structure. Electronic states of a pair of vacancies are investigated in $(4 \times 4)$ supercells by using $(3 \times 3 \times 1) k$-point sampling by including spin polarizations. The formation energy of a cation-anion pair of vacancies is calculated using the expression $E_{v}=E_{T}[\mathrm{Ga}(\mathrm{Al}) \mathrm{N}+\mathrm{V}]-\frac{m-1}{m} E_{T}[\mathrm{Ga}(\mathrm{Al}) \mathrm{N}]$, in terms of the total energy of SL $s o-\mathrm{GaN}$ and $s o-\mathrm{AlN}$ structures containing a pair of cation-anion vacancies and that of the bare $s o$-structures containing $m$ cation-anion pairs. All calculations are performed in a $(4 \times 4)$ supercell with full structure optimization. Here, we considered two situations for each $s o$-structure, where either one cation and one anion at different locations of the supercell are removed or a single cation-anion bond is removed. In both cases, charge neutrality is maintained.

The formation energy of a neutral Ga-N (Al-N) vacancy is calculated to be $8.66 \mathrm{eV}(11.06 \mathrm{eV})$, if single $\mathrm{Ga}(\mathrm{Al})$ and $\mathrm{N}$ vacancies occur in different bonds. On the other hand, the formation energy of a pair of Ga-N (Al-N) vacancy created by removing nearest $\mathrm{Ga}(\mathrm{Al})$ and $\mathrm{N}$ atoms, which form a Ga-N (Al-N) bond from a square of a SL $s o-G a N(s o-A l N)$ is calculated to be $5.18 \mathrm{eV}(7.22 \mathrm{eV})$. If a similar pair of 

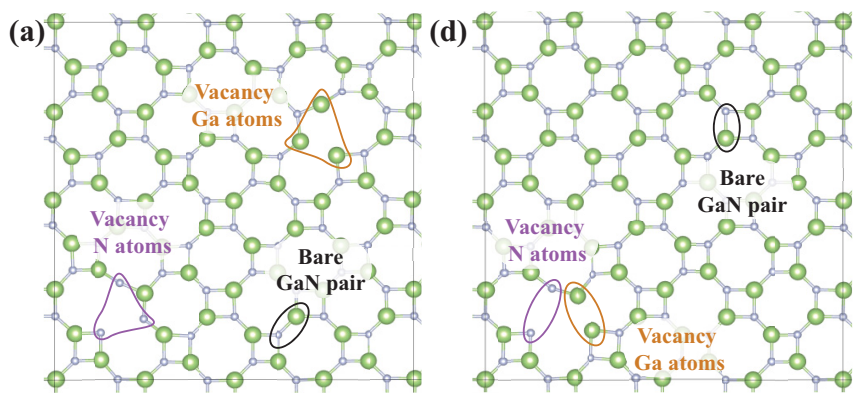

(b)

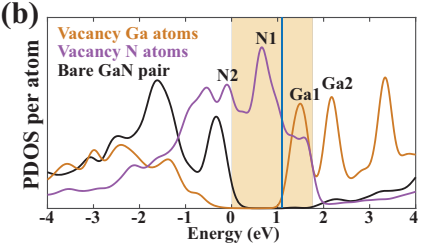

(c)
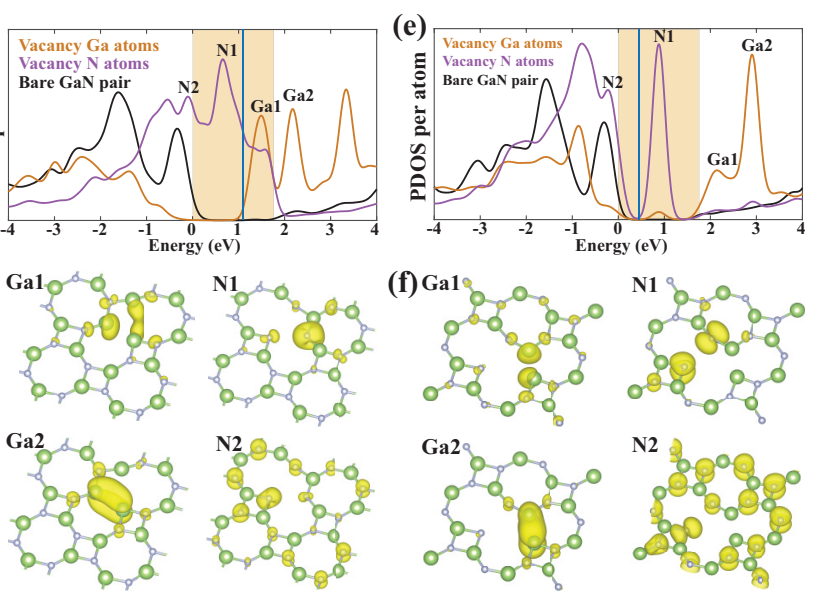

FIG. 8. Analysis of projected densities of states and isosurfaces of the charge densities of the states, which give rise to specific peaks near the fundamental band gap upon the creation of a pair of $\mathrm{Ga}-\mathrm{N}$ vacancies in SL $s o-G a N$. (a) $\mathrm{Ga}$ and $\mathrm{N}$ vacancies are created in different bonds. A Ga-N bond far from these vacancies is indicated. (b) Densities of states projected on atoms surrounding $\mathrm{Ga}$ and $\mathrm{N}$ vacancies. The density of states projected to a Ga-N bond far from $\mathrm{Ga}$ and $\mathrm{Al}$ vacancies mimics the density of states of the bare SL so-GaN and its fundamental band gap (shaded) relative to the Fermi level. Peaks near the fundamental band gap of the bare $s o-\mathrm{GaN}$ are indicated. The zero of energy is set to the common Fermi level of vacancy + so-GaN. (c) Isosurfaces of the charge densities of the states forming peaks near the fundamental band gap. (d)-(f) Same for a pair of $\mathrm{Ga}$ and $\mathrm{N}$ vacancies, which is created by removing a Ga-N bond.

Ga-N (Al-N) vacancy is created in a cation-anion bond of octagon of a SL $s o-\mathrm{GaN}(s o-\mathrm{AlN})$, the formation energy is calculated to be $5.65 \mathrm{eV}(7.09 \mathrm{eV})$. These results indicate that the formation energy of adjacent cation-anion vacancy depends on its location. Also, when cation and anion vacancies are not adjacent, the formation energy becomes larger since more bonds ought to be broken compared to the adjacent cationanion vacancy.

$\mathrm{SL} s o-\mathrm{GaN}$ or $s o$-AlN acquires a permanent magnetic moment of $2.0 \mu_{B}$ through the formation of a cation-anion vacancy. This way, SL $s o$-structure can attain large and local magnetic moments by creating a large amount of cation-anion vacancies in a domain. Not only magnetic properties, but also electronic structure of SL $s o$-structure are modified by forming a cation-anion vacancy. For example, in Figs. 8(a)-8(c) the Ga and $\mathrm{N}$ vacancies occurring in different bonds gives rise to the peaks in the fundamental band gap. The strongest peak $\mathrm{N}_{1}$ occurs in the midgap and is due to filled states localized at $\mathrm{N}$ atoms nearest to Ga vacancy. The peak of empty states $\mathrm{Ga}_{1}$,
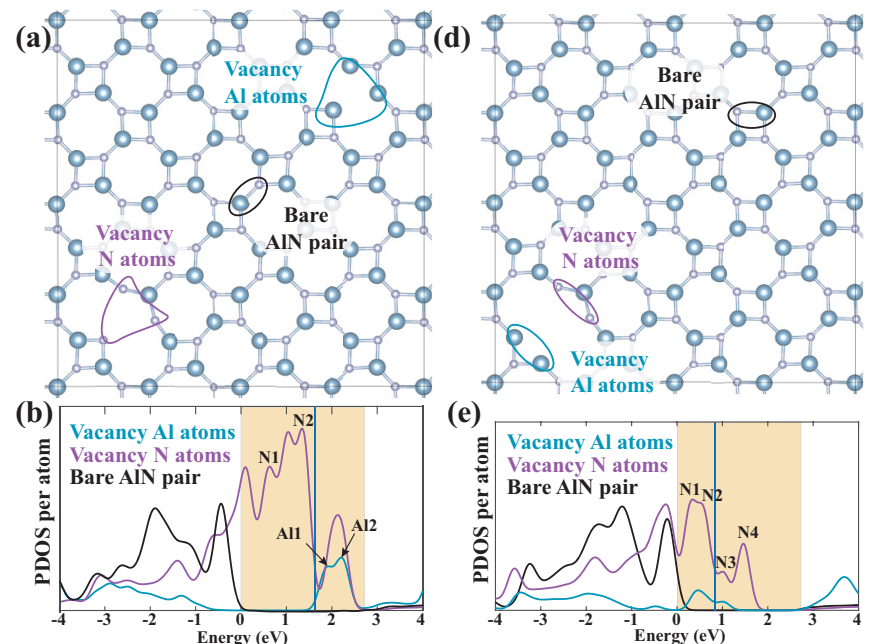

(e)

(c)
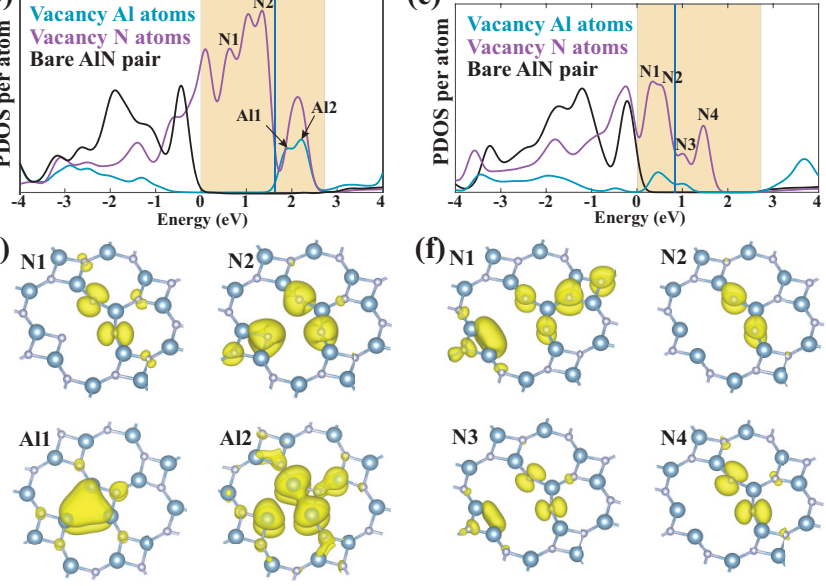

FIG. 9. Analysis of projected densities of states and isosurfaces of the charge densities of the states, which give rise to specific peaks near the fundamental band gap upon the creation of a pair of Al-N vacancies in SL so-AlN. (a)-(f) Same as in Fig. 8 presented for so-GaN.

which are localized at $\mathrm{Ga}$ atoms nearest to $\mathrm{N}$ vacancy, occurs near the conduction band edge. In the case of a pair of adjacent Ga-N vacancies in Figs. 8(d)-8(f), the $\mathrm{N}_{1}$ peak of empty states, which is localized at $\mathrm{N}$ atoms nearest to the Ga vacancy, occurs above the Fermi level. In $s o$-AIN structure gap states localized at $\mathrm{N}$ atoms nearest to the $\mathrm{Al}$ vacancy are occupied, whereas states localized at $\mathrm{Al}$ atoms nearest to $\mathrm{N}$ vacancy in a different Al-N bond are unoccupied, as seen in Fig. 9. However, when $\mathrm{Al}$ and $\mathrm{N}$ vacancies are adjacent and occur at one of the sides of a square, all gap states are localized at $\mathrm{N}$ atoms nearest to Al vacancy; but, no gap states occur localized at $\mathrm{Al}$ atoms nearest to $\mathrm{N}$ vacancy. Occurrences of localized gap states in diverse energies in the band gap have a close bearing on the cation-anion vacancy having different bond angles and hence $s p^{x}$ hybridization. Briefly, cation-anion neutral vacancies of $s o$-structures give rise to localized gap states, which modify the electronic structure significantly.

\section{CONCLUSIONS}

While 2D hexagonal structures of $\mathrm{AlN}$ and GaN are gaining importance following the synthesis of their ultrathin layers, we investigated single-layer, square-octagon structures of $\mathrm{GaN}$ and AIN. To ensure their stability at room temperatures, we first carried out an extensive analysis of dynamical and thermal stability using ab initio phonon and finite-temperature molecular dynamic calculations, which indicated that these free-standing structures remain stable at high temperature. Our 
study of mechanical properties provided evidences that they are robust. We analyzed the symmetries of phonon modes and revealed the Raman and infrared active modes as fingerprints of these structure. We predict a strong directionality in the calculated elastic constants. In particular, these structures become stiffer under the tensile stress along the diagonal of the unit cell. Both $s o-\mathrm{GaN}$ and $s o$-AlN structures are wide-band-gap semiconductors with indirect fundamental band gap. Under uniaxial and biaxial strain, these band gaps reduce and eventually are closed with increasing strain. The electronic structure can also be modified by forming bilayers, multilayers, and 3D layered structures through stacking of planar $s o$-structures. We considered three different types of stacking geometries and investigated their energetics, stability, and electronic properties. As a most remarkable feature of these multilayer $s o$-structures we found the vertical cationanion bonds between adjacent layers. These bonds are weaker than the in-plane cation-anion bonds, but contribute to the van der Waals interaction in the binding of layers. The planar geometries of the constituent layers are buckled in multilayer structures. Moreover, because of the electronic coupling between layers, the effect of multilayer formation on the electronic structure is stronger than those in vertical van der Waals solids. In this respect, bilayers and multilayers constructed by the stacking of planar, single-layer GaN and AlN differ from van der Waals solids. For specific stacking geometries, we predict that stable 3D layered structures can be constructed from single layers, which can have direct band gaps with cohesive energies slightly smaller than that of $3 \mathrm{D}$ wurtzite structures known to correspond to the global minimum.

Finally, we investigated the effects of adatoms adsorbed to $s o$-structures and creation of the vacancy of a cation-anion on electronic and magnetic properties. Specific adatoms can modify the electronic structure through their localized gap states and can acquire local magnetic moment. As a result, by decoration of adatoms and doping, $s o$-structures can be functionalized to present new physical properties. As a point defect, neutral vacancy of a cation-anion pair gives rise to diverse electronic structure and local magnetic moment. In particular, depending on the locations of cation and anion vacancies, localized gap states occur in different energies in the band gap. This situation stems from different bond angles and resulting $s p^{x}$ hybridization in the square-octagon rings.

In conclusion, we showed that square-octagon structures of nitride semiconductors are stable and robust and offer several properties suitable for 2D electronics. They can be functionalized by adatoms and vacancies, and they can construct multilayers to form new nanostructures. In plane and vertical heterostructures of $s o-\mathrm{GaN}$ and $s o$-AlN one can form multiple quantum wells and resonant tunneling devices.

\section{ACKNOWLEDGMENTS}

The computational resources are provided by TUBITAK ULAKBIM, High Performance and Grid Computing Center (TR-Grid e-Infrastructure) and the National Center for High Performance Computing of Turkey (UHeM) under Grant No. 5003622015. This work was supported by the Scientific and Technological Research Council of Turkey (TUBITAK) under Project No. 115F088. E.D. acknowledges the financial support from the Turkish Academy of Sciences within Outstanding Young Scientists Award Program (TUBA-GEBIP). S. Ciraci acknowledges financial support from the Academy of Sciences of Turkey (TUBA). S.C. acknowledges support from Bilim Akademisi-The Science Academy, Turkey, under the BAGEP program.
[1] S. Nakamura, M. Tagashi, and S. Masayuki, Appl. Phys. Lett. 64, 1687 (1994); S. Nakamura, Rev. Mod. Phys. 87, 1139 (2015).

[2] H. Morkoc, Handbook of Nitride Semiconductors and Devices (Wiley, Weinheim, 2008), Vol. 1.

[3] K. S. Novoselov, A. K. Geim, S. V. Morozov, D. Jiang, Y. Zhang, S. V. Dubonos, I. V. Grigorieva, and A. A. Firsov, Science 306, 666 (2004).

[4] E. Durgun, S. Tongay, and S. Ciraci, Phys. Rev. B 72, 075420 (2005).

[5] S. Cahangirov, M. Topsakal, E. Akturk, H. Sahin, and S. Ciraci, Phys. Rev. Lett. 102, 236804 (2009).

[6] H. Sahin, S. Cahangirov, M. Topsakal, E. Bekaroglu, E. Akturk, R. T. Senger, and S. Ciraci, Phys. Rev. B 80, 155453 (2009).

[7] M. Topsakal, E. Aktŭrk, and S. Ciraci, Phys. Rev. B 79, 115442 (2009).

[8] E. Bekaroglu, M. Topsakal, S. Cahangirov, and S. Ciraci, Phys. Rev. B 81, 075433 (2010).

[9] M. Topsakal, S. Cahangirov, E. Bekaroglu, and S. Ciraci, Phys. Rev. B 80, 235119 (2009).

[10] P. Vogt, P. De Padova, C. Quaresima, J. Avila, E. Frantzeskakis, M. C. Asensio, A. Resta, B. Ealet, and G. Le Lay, Phys. Rev. Lett. 108, 155501 (2012).
[11] M. E. Dávila and G. LeLay, Sci. Rep. 6, 20714 (2016).

[12] S. Cahangirov, M. Audiffred, P. Tang, A. Iacomino, W. Duan, G. Merino, and A. Rubio, Phys. Rev. B 88, 035432 (2013).

[13] C. L. Freeman, F. Claeyssens, N. L. Allan, and J. H. Harding, Phys. Rev. Lett. 96, 066102 (2006).

[14] Y. Wang and S. Shi, Solid State Commun. 150, 1473 (2010).

[15] Y. Ma, Y. Dai, M. Guo. C. Niu, L. Yu, and B. Huang, Appl. Surf. Sci. 257, 7845 (2011).

[16] A. K. Singh and R. G. Hennig, Appl. Phys. Lett. 105, 051604 (2014).

[17] A. K. Singh, H. L. Zhuang, and R. G. Hennig, Phys. Rev. B 89 , 245431 (2014).

[18] Q. Chen, H. Hu, X. Chen, and J. Wang, Appl. Phys. Lett. 98, 053102 (2011)

[19] D. Kecik, C. Bacaksiz, R. T. Senger, and E. Durgun, Phys. Rev. B 92, 165408 (2015).

[20] C. Bacaksiz, H. Sahin, H. D. Ozaydin, S. Horzum, R. T. Senger, and F. M. Peeters, Phys. Rev. B 91, 085430 (2015).

[21] A. Onen, D. Kecik, E. Durgun, and S. Ciraci, Phys. Rev. B 93, 085431 (2016).

[22] Y. Gao, T. Yayama, and S. Okada, Appl. Phys. Express 9, 095201 (2016). 
[23] A. V. Kolobov, P. Fons, J. Tominaga, B. Hyot, and B. Andre, Nano Lett. 16, 4849 (2016).

[24] D. Wu, M. G. Lagally, and F. Liu, Phys. Rev. Lett. 107, 236101 (2011).

[25] H. L. Zhuang, A. K. Singh, and R. G. Hennig, Phys. Rev. B 87, 165415 (2013).

[26] D. V. Fakhrabad, N. Shahtahmasebi, and M. Ashhadi, Superlattices Microstruct. 79, 38 (2015).

[27] A. Onen, D. Kecik, E. Durgun, and S. Ciraci, Phys. Rev. B 95, 155435 (2017).

[28] P. Tsipas, S. Kassavetis, D. Tsoutsou, E. Xenogiannopoulou, E. Golias, S. A. Giamini, C. Grazianetti, D. Chiappe, A. Molle, M. Fanciulli, and A. Dimoulas, Appl. Phys. Lett. 103, 251605 (2013).

[29] Z. Y. Al Balushi, K. Wang, R. K. Ghosh, R. A. Vila, S. M. Eichfeld, J. D. Caldwell, X. Qin, Y.-C. Lin, P. A. DeSario, G. Stone et al., Nat. Mater. 15, 1166 (2016).

[30] R. H. Baughman and H. Eckhardt, J. Chem. Phys. 87, 6687 (1987).

[31] A. Hirsch, Nat. Mater. 9, 868 (2010).

[32] F. Diederics, Nature (London) 369, 199 (1994).

[33] W. Li, M. Guo, G. Zhang, and Y.-W. Zhang, Phys. Rev. B 89, 205402 (2014).

[34] V. O. Özçelik and S. Ciraci, J. Phys. Chem. C 117, 2175 (2013).

[35] F. Ersan, E. Aktürk, and S. Ciraci, J. Phys. Chem. C 120, 14345 (2016).

[36] D. C. Camacho-Mojica and F. Lopez-Urias, Sci. Rep. 5, 17902 (2015).

[37] H. Zhang, F.-S. Meng, and Y.-B. Wu, Solid State Commun. 250, 18 (2017).

[38] P. A. Brown and K. L. Shuford, Nanoscale 8, 19287 (2016).

[39] P. E. Blöchl, Phys. Rev. B 50, 17953 (1994).

[40] G. Kresse and J. Hafner, Phys. Rev. B 49, 14251 (1994).

[41] G. Kresse and J. Furthmüller, Comput. Mater. Sci. 6, 15 (1996).
[42] J. P. Perdew, K. Burke, and M. Ernzerhof, Phys. Rev. Lett. 77, 3865 (1996).

[43] H. J. Monkhorst and J. D. Pack, Phys. Rev. B 13, 5188 (1976).

[44] D. Alfe, Comput. Phys. Commun. 180, 2622 (2009).

[45] S. Demirci, N. Avazlı, E. Durgun, and S. Cahangirov, Phys. Rev. B 95, 115409 (2017).

[46] M. Topsakal, S. Cahangirov, and S. Ciraci, Appl. Phys. Lett. 96, 091912 (2010).

[47] Q. Peng, C. Liang, W. Ji, and S. De, Appl. Phys. A: Mater. Sci. Process. 113, 483 (2013).

[48] D. Xu, H. He, R. Pandey, and S. P. Karna, J. Phys.: Condens. Matter 25, 345302 (2013).

[49] C. Attaccalite, A. Nguer, E. Cannuccia, and M. Gruning, Phys. Chem. Chem. Phys. 17, 9533 (2015).

[50] R. B. dos Santos, F. de Brito Mota, R. Rivelino, A. KakanakovaGeorgieva, and G. K. Gueorguiev, Nanotechnology 27, 145601 (2016).

[51] C.-J. Tong, H. Zhang, Y.-N. Zhang, H. Liu, and L.-M. Liu, J. Mater. Chem. A 2, 17971 (2014).

[52] M. S. Prete, A. M. Conte, P. Gori, F. Bechstedt, and O. Pulci, Appl. Phys. Lett. 110, 012103 (2017).

[53] J. Paier, M. Marsman, K. Hummer, G. Kresse, I. C. Gerber, and J. G. Angyan, J. Chem. Phys. 124, 154709 (2006).

[54] S. Grimme, J. Antony, S. Ehrlich, and S. Krieg, J. Chem. Phys. 132, 154104 (2010).

[55] S. Grimme, S. Ehrlich, and L. Goerigk, J. Comput. Chem. 32, 1456 (2011).

[56] S. Grimme, J. Comput. Chem. 27, 1787 (2006).

[57] A. Tkatchenko and M. Scheffler, Phys. Rev. Lett. 102, 073005 (2009).

[58] O. Ü. Aktürk, E. Aktürk, and S. Ciraci, Phys. Rev. B 93, 035450 (2016).

[59] S. Ciraci, A. Baratoff, and I. P. Batra, Phys. Rev. B 42, 7618 (1990). 